\title{
Lateral lumbar interbody fusion after reduction using the percutaneous pedicle screw system in the lateral position for Meyerding grade II spondylolisthesis: a preliminary report of a new lumbar reconstruction strategy
}

\author{
Masanari Takami* (D, Ryo Taiji, Motohiro Okada, Akihito Minamide, Hiroshi Hashizume and Hiroshi Yamada
}

\begin{abstract}
Background: Utilization of a cage with a large footprint in lateral lumbar interbody fusion (LLIF) for the treatment of spondylolisthesis leads to a high fusion rate and neurological improvement owing to the indirect decompression effect and excellent alignment correction. However, if an interbody space is too narrow for insertion of an LLIF cage for cases of spondylolisthesis of Meyerding grade II or higher, LLIF cannot be used. Therefore, we developed a novel strategy, LLIF after reduction by the percutaneous pedicle screw (PPS) insertion system in the lateral position (LIFARL), for surgeons to perform accurate and safe LLIF with PPS in patients with such pathology. This study aimed to introduce the new surgical strategy and to present preliminary clinical and radiological results of patients with spondylolisthesis of Meyerding grade II.
\end{abstract}

Methods: Six consecutive patients (four men and two women; mean age, 72.7 years-old; mean follow-up period, 15.3 months) with $L 4$ spondylolisthesis of Meyerding grade II were included. Regarding the surgical procedure, first, PPSs were inserted into the L4 and L5 vertebrae fluoroscopically, and both rods were placed in the lateral position. The $L 5$ set screws were fixed tightly, and the $L 4$ side of the rod was floated. Second, the $L 4$ vertebra was reduced by fastening the $L 4$ set screws so that they expanded the anteroposterior width of the interbody space. At that time, the $L 4$ set screws were not fully tightened to the rods to prevent the endplate injury. Finally, the LLIF procedure was started. After inserting the cage, a compression force was added to the PPSs, and the L4 set screws were completely fastened.

Results: The mean operative time was $183 \mathrm{~min}$, and the mean blood loss was $90.8 \mathrm{~mL}$. All cages were positioned properly. Visual analog scale score and Oswestry disability index improved postoperatively. Bone union was observed using computed tomography 12 months after surgery.

(Continued on next page)

\footnotetext{
*Correspondence: takami@wakayama-med.ac.jp

Department of Orthopaedic Surgery, Wakayama Medical University, 811-1 Kimiidera, Wakayama 641-8510, Japan
} 
(Continued from previous page)

Conclusion: For cases with difficulty in LLIF cage insertion for Meyerding grade II spondylolisthesis due to the narrow anteroposterior width of interbody space, LIFARL is an option to achieve LLIF combined with posterior PPS accurately and safely.

Trial registration: UMIN-Clinical Trials Registry, UMIN000040268, Registered 29 April 2020, https://upload.umin.ac.jp/ cgi-open-bin/ctr/ctr_view.cgi?recptno=R000045938

Keywords: Meyerding grade II spondylolisthesis, Lateral lumbar interbody fusion, Percutaneous pedicle screw, Minimally invasive spine surgery, Lateral position, New surgical technique

\section{Background}

Spondylolisthesis (SL) is a common disease, and surgical treatment is sometimes selected when patients complain of leg or back pain. Posterior lumbar interbody fusion (PLIF) and transforaminal lumbar interbody fusion are conventional surgical methods for treating SL [1]. Lateral lumbar interbody fusion (LLIF) has been developed recently [2]. Subsequently, the use of minimally invasive spinal surgery involving LLIF combined with posterior percutaneous pedicle screw (PPS) fixation [3] for degenerative lumbar disease was reported. Utilization of a cage with a large footprint in LLIF leads to a high fusion rate [4-6] and neurological improvement because of the indirect decompression effect and excellent alignment correction $[2,7]$. This surgical strategy has been adopted in patients with $\mathrm{SL}$, and good clinical and radiological results have been demonstrated [8-17]. However, if patients, especially Asians with small physiques, have grade II or higher SL according to the Meyerding classification [18], there is a disadvantage in that it is difficult to insert the large cage into a narrow interbody space (Fig. 1a, b). Therefore, we developed a novel surgical strategy of lateral lumbar interbody fusion after reduction by the posterior percutaneous pedicle screw system in the lateral position (LIFARL) for such patients. To the best of our knowledge, this is the first description of such a strategy. The current study aimed to introduce our novel surgical strategy and present preliminary clinical and radiological results.

\section{Methods \\ Study design and patients}

The study design was approved by the institutional review board of our university before the initiation of the study. The patients or their family members were informed that the patient data would be submitted for publication, and their written consent was obtained.

We performed extreme lateral interbody fusion (XLIF ${ }^{\oplus}$, NuVasive, San Diego, CA, USA) combined with PPS (Reline MAS system ${ }^{\circ}, \mathrm{NuVasive)}$ in the lateral position for 15 patients with lumbar diseases since June 2018 and developed our novel strategy and have been using it since September 2018. Six consecutive patients (four men and two women) who underwent fusion surgery by this method in our institute for Meyerding grade II SL with leg or low back pain were included in this study.

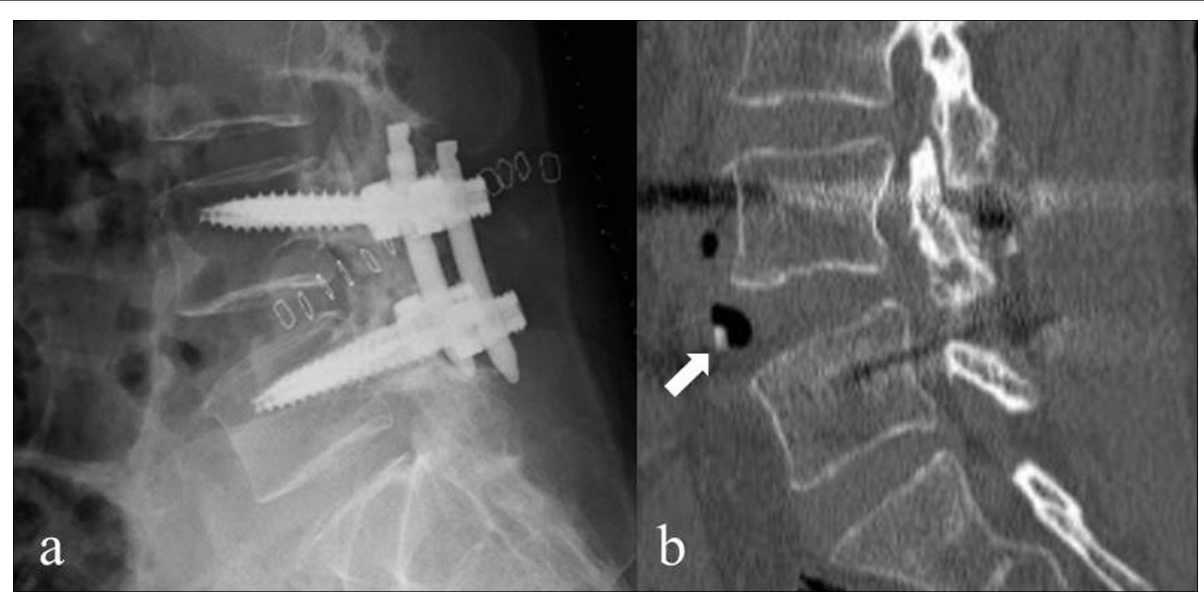

Fig. 1 Failure of extreme lateral interbody fusion (XLIF) for a patient with L4 spondylolisthesis. L4-5 fusion surgery, including XLIF, is planned for a 58-year-old woman with L4 spondylolisthesis. However, the anterior longitudinal ligament (ALL) is injured during the XLIF procedure; hence, XLIF is abandoned because it is impossible to fix a cage. Postoperative radiography reveals that the interbody space at the $L 4 / 5$ level expanded abnormally $\mathbf{a}$. After surgery, a bony endplate fracture (white arrow) associated with the ALL tear is detected on computed tomography $\mathbf{b}$ 


\section{Surgical procedure}

The following new surgical strategy was described as a case of L4-5 fusion surgery for SL of the L4 vertebra. After placing the patient in the lateral position, XLIF and posterior PPS were used simultaneously. First, PPSs were inserted into the L4 and L5 vertebrae fluoroscopically (Fig. 2a), and rods on both sides of appropriate lengths were placed. The final fixation of the L5 set screws on both sides was done, and the L4 side of the rod was floated. Next, the L4 vertebra was reduced to some extent by fastening the L4 set screws on both sides through the extender such that they expanded to the anteroposterior width of the interbody space. Using this operation, Meyerding grade changed from II to I, and the cage could be safely inserted. At this time, the L4 set screws were not fully tightened to the rods (i.e., movement between the L4 PPSs and rods remained). The anteroposterior space in which the XLIF cage would be inserted was expanded by this reduction. Finally, XLIF was started, and disc curettage was conducted after the retractor was placed in the lateral position (Fig. 2b). At this time, motion between the L4 and L5 vertebrae was confirmed under fluoroscopy (Fig. 2c, d), which helped to prevent endplate injury upon insertion of the XLIF cage. After reducing the $\mathrm{L} 4$ vertebra again by tightening the L4 set screws if necessary, the XLIF cage was inserted. Reduction of the L4 vertebra was never performed after cage insertion to prevent endplate injury. After the bending of the surgical table was restored, a compression force was added to the PPSs, and both L4 set screws were completely fastened to finish the surgical procedures.

\section{Evaluation of clinical variables}

The operative time and estimated blood loss were evaluated. Complications and radiological findings were evaluated. Measurements of slippage, treated intervertebral heights, evaluation of endplate injury during surgery, malposition of the pedicle screws and screw loosening, inserted cage position in the interbody space, bone union based on the Proietti classification [4], and cage subsidence (over $2 \mathrm{~mm}$ ) were included in the radiological investigations. Regarding radiological evaluations, bone union was assessed using computed tomography (CT) 12 months after surgery, and the other factors were evaluated by X-rays at the final observation. Regarding clinical results, the Oswestry disability index (ODI) and low back and leg pain based on visual analog scale (VAS) score were evaluated at the final observation.

\section{Results}

\section{Clinical and radiological outcomes}

Mean age was 72.7 years (range, $52-83$ years), and the mean follow-up period was 15.3 months (Table 1).

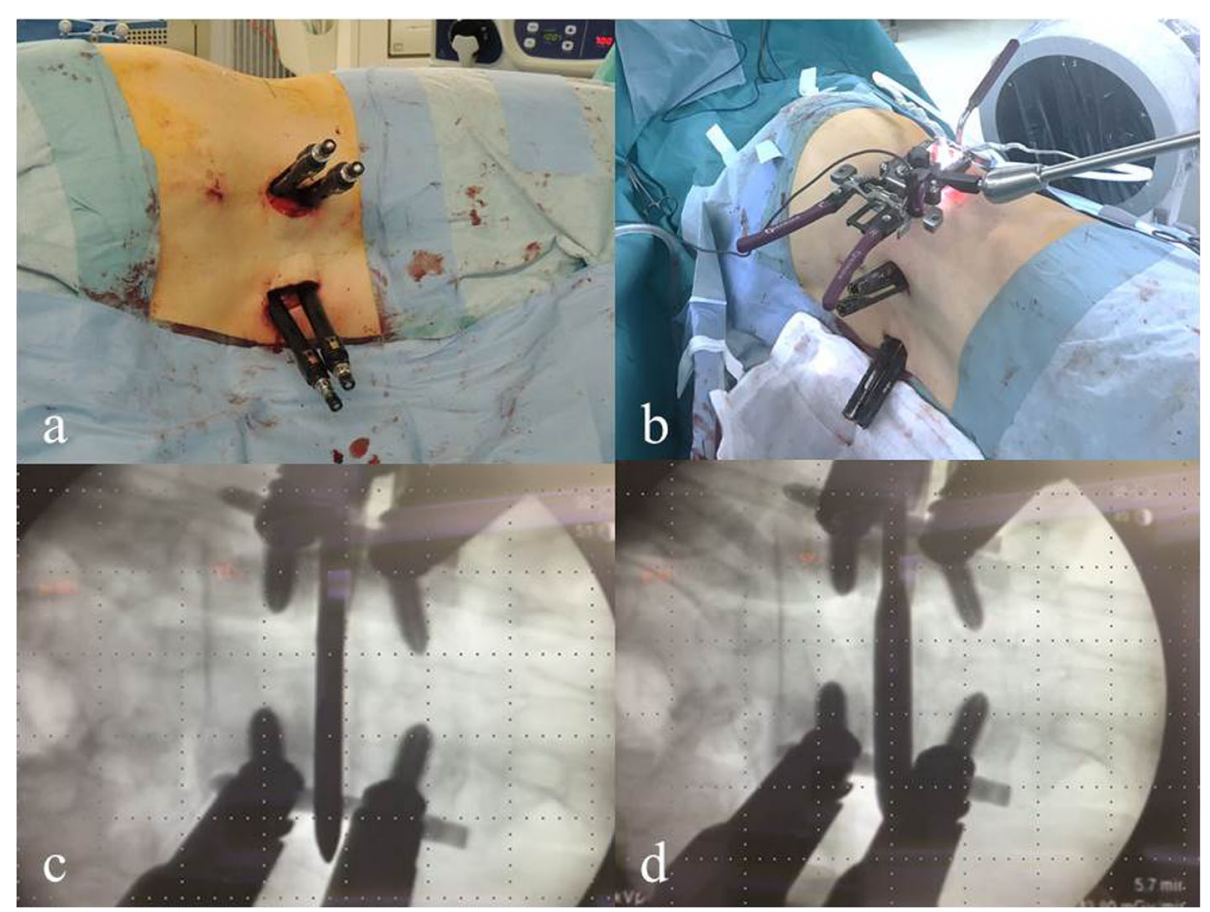

Fig. 2 Intraoperative image in the lateral position and fluoroscopic imaging. Surgery is started by inserting the percutaneous pedicle screws with the patient in the lateral position a. Extreme lateral interbody fusion is performed after reduction by using the percutaneous pedicle screws system $\mathbf{b}$. During extreme lateral interbody fusion, fluoroscopy is used to insert the XLIF Paddle Sizer ${ }^{\circledR} \mathbf{c}$, and when the Paddle Sizer is rotated, there is some movement between the $L 4$ and $L 5$ vertebrae $\mathbf{d}$. This helps to prevent endplate injury during cage insertion 
Table 1 Clinical characteristics of patients

\begin{tabular}{lllll}
\hline Case & Level of Spodylolisthesis & Meyerding classification & Fusion level & Follow-up period (mos.) \\
\hline 1 & $\llcorner 4$ & Grade II & $\llcorner 4-5$ & 19 \\
2 & $\llcorner 4$ & Grade II & $\llcorner 4-5$ & 19 \\
3 & Grade II & $\llcorner 3-5$ & 18 \\
4 & Grade II & $\llcorner 4-5$ & 14 \\
5 & $\llcorner 4$ & Grade II & $\llcorner 4-5$ & 12 \\
6 & $\llcorner 4$ & Grade II & $L 3-5$ & 10 \\
\hline
\end{tabular}

The following data are presented as mean \pm standard deviation. The mean operative time was $183.0 \pm 40.3$ min, and the mean estimated blood loss was $90.8 \pm 68.2$ $\mathrm{mL}$ (Table 2). No complications occurred except in one patient who developed temporary quadriceps weakness for 2 months. Regarding the radiological evaluations, the average slippage amount improved from $12.7 \pm 0.8 \mathrm{~mm}$ preoperatively to $3.7 \pm 2.8 \mathrm{~mm}$ at the final observation. The percentage of slippage improved from $33.2 \pm 2.4 \%$ preoperatively to $9.5 \pm 7.3 \%$ at the final observation. The height between the vertebrae improved from $3.3 \pm 2.7$ $\mathrm{mm}$ preoperatively to $8.7 \pm 0.5 \mathrm{~mm}$ at the final observation. There were no cases of endplate injury during surgery, malposition of the pedicle screws, or screw loosening. All cages were inserted properly (Fig. 3). Regarding bone union, type IV (bone bridges inside one of the two internal spaces of the cage and on one side outside of the cage), and type $\mathrm{V}$ (bone bridges inside one of the two internal spaces of the cage and on both sides outside of cage) according to the Proietti classification was found in three and three cases, respectively. No cage subsidence was found. The mean ODI improved from $44.2 \pm 4.8$ preoperatively to $23.7 \pm 15.3$ postoperatively. The mean VAS score for low back pain improved from $67.5 \pm 7.7 \mathrm{~mm}$ preoperatively to $21.8 \pm 17.8 \mathrm{~mm}$ postoperatively, and the mean VAS score for leg pain improved from $65.8 \pm 18.3 \mathrm{~mm}$ preoperatively to $19.8 \pm 17.8 \mathrm{~mm}$ postoperatively. In case 2, pre-ODI was nearly equal to post-ODI because it was evaluated immediately before total knee arthroplasty due to his right knee osteoarthritis. However, his low back and leg pain, as indicated by VAS, have improved. No patient required additional decompression surgery postoperatively.

\section{Representative case}

A 64-year-old man was diagnosed with degenerative SL of the L4 vertebra with instability and simple lumbar spinal stenosis at the L3/4 levels (Fig. 4a, b). Spinal fusion surgery at the L3-5 levels was performed (Fig. 5 \& $6 \mathrm{a}, \mathrm{b}, \mathrm{a}, \mathrm{b})$. The operative time was $163 \mathrm{~min}$, and blood loss was $30 \mathrm{~mL}$. At 18 months postoperatively, the patient's lumbar and leg pains disappeared. Postoperative magnetic resonance imaging revealed that the spinal canal had expanded enough by indirect decompression. Type IV bone union according to the Proietti classification was observed using $\mathrm{CT}$ at 12 months postoperatively. No implant-related complications occurred at the final observation.

\section{Discussion}

This is the first description of the novel strategy to perform XLIF combined with posterior PPS for patients with Meyerding grade II SL. Conventional XLIF combined with PPS procedure for such patients is

Table 2 Clinical and radiological results. Regarding clinical results, measurements of vertebral slippage and disc heights using lateral lumbar radiography before and after surgical correction, and low back and leg pain using VAS score and ODI before surgical treatment and at the latest clinical follow-up were performed. Bone union pattern by Proietti classification was evaluated 12 months after surgery, and cage subsidence was examined at the final observation

\begin{tabular}{|c|c|c|c|c|c|c|c|c|c|c|c|c|c|c|c|}
\hline \multirow[t]{2}{*}{ Case } & \multirow{2}{*}{$\begin{array}{l}\text { Op. } \\
\text { time } \\
\text { (min.) }\end{array}$} & \multirow[t]{2}{*}{$\begin{array}{l}\text { EBL } \\
\text { (g) }\end{array}$} & \multirow[t]{2}{*}{$\begin{array}{l}\text { APW } \\
(\mathrm{mm})\end{array}$} & \multicolumn{2}{|c|}{ Slippage (mm) (\%) } & \multicolumn{2}{|c|}{$\mathrm{DH}(\mathrm{mm})$} & \multicolumn{2}{|c|}{$\begin{array}{l}\text { Low back pain in } \\
\text { VAS }\end{array}$} & \multicolumn{2}{|c|}{ Leg pain in VAS } & \multicolumn{2}{|l|}{ ODI } & \multirow{2}{*}{$\begin{array}{l}\text { Bone fusion } \\
\text { by Proietti } \\
\text { classification }\end{array}$} & \multirow[t]{2}{*}{$\begin{array}{l}\text { Cage } \\
\text { subsidence }\end{array}$} \\
\hline & & & & Pre-op & Post-op & Pre-op & Post op & Pre-op & Post-op & Pre-op & Post-op & Pre-op & Post-op & & \\
\hline 1 & 203 & 130 & 25 & $12(31.6 \%)$ & $0(0.0 \%)$ & 1 & 8 & 70 & 22 & 50 & 23 & 46 & 26 & IV & No \\
\hline 2 & 202 & 205 & 24 & $12(30.0 \%)$ & $1(2.5 \%)$ & 6 & 9 & 71 & 18 & 100 & 15 & 47 & $51^{*}$ & IV & No \\
\hline 3 & 163 & 30 & 21 & $13(34.2 \%)$ & $3(7.9 \%)$ & 4 & 9 & 73 & 10 & 72 & 9 & 48 & 10 & IV & No \\
\hline 4 & 162 & 30 & 20 & 13 (34.2\%) & $6(14.3 \%)$ & 1 & 9 & 73 & 10 & 60 & 20 & 48 & 9 & V & No \\
\hline 5 & 127 & 55 & 17 & $12(32.4 \%)$ & 7 (18.9\%) & 7 & 8 & 53 & 57 & 55 & 52 & 38 & 26 & V & No \\
\hline 6 & 241 & 95 & 21 & $14(36.8 \%)$ & $5(13.1 \%)$ & 1 & 8 & 65 & 14 & 58 & 0 & 38 & 20 & $\mathrm{~V}+$ & No \\
\hline
\end{tabular}

EBS Estimated blood loss, APW Anteroposterior width of interbody space at spondylolisthesis preoperatively, $\mathrm{DH}$ Disc height, ODI Oswestry disability index, ${ }^{*}$ This item was evaluated immediately before total knee arthroplasty due to his right knee osteoarthritis, ${ }^{\dagger}$ This evaluation was performed 10 months after surgery 

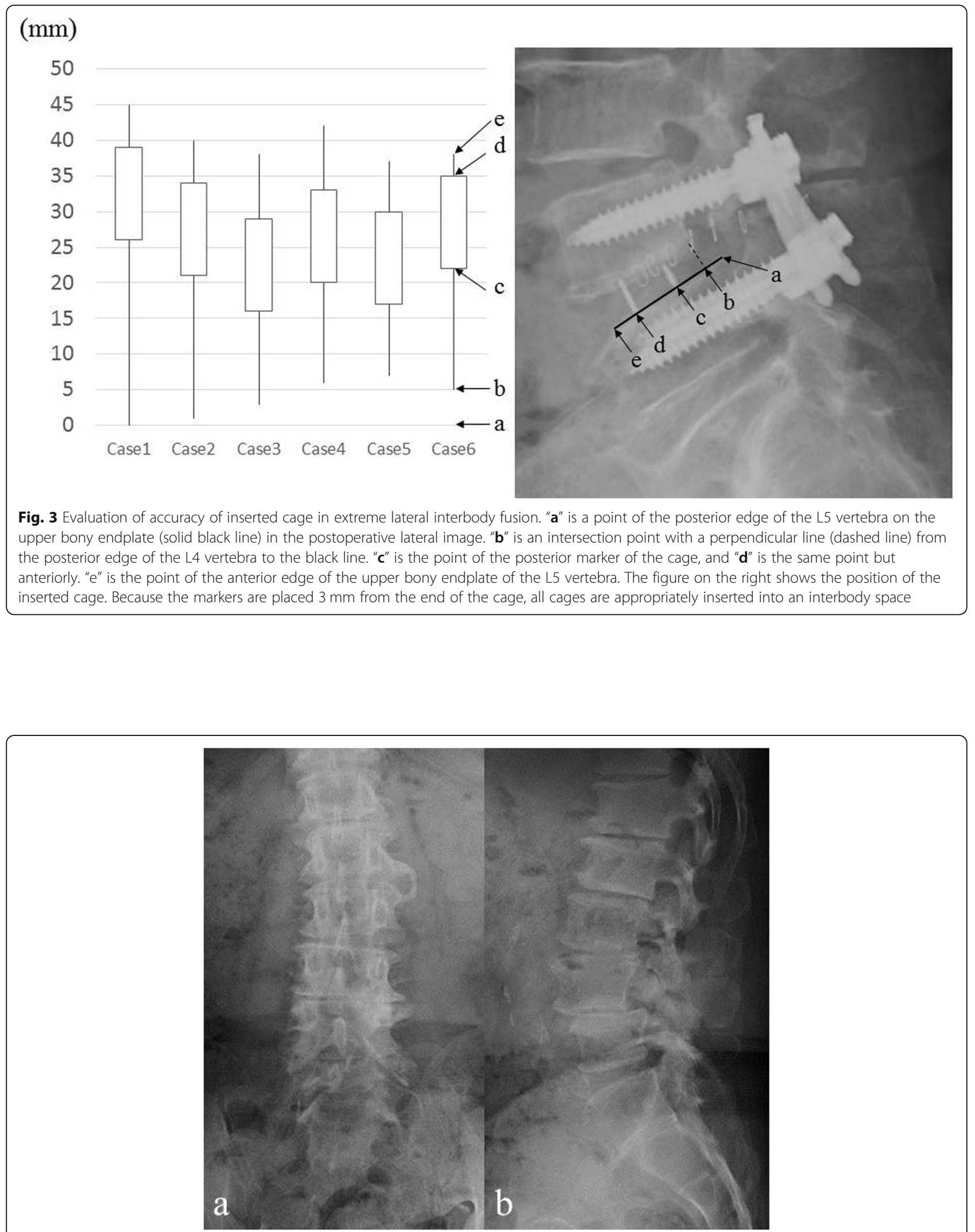

Fig. 4 Preoperative radiographic imaging. Preoperative radiographic coronal image a and radiographic lateral image $\mathbf{b}$ showing Meyerding grade II spondylolisthesis 


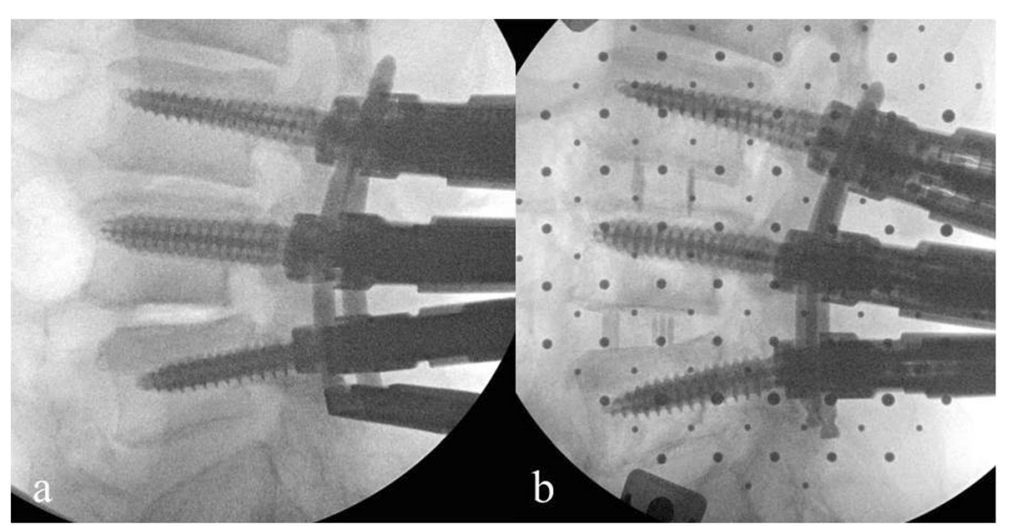

Fig. 5 Intraoperative fluoroscopic imaging during slipped vertebra reduction prior to extreme lateral interbody fusion. Fluoroscopic lateral image after reduction is performed by pulling the rods backward, and the extender shows an increase of anteroposterior interbody space at the $L 4 / 5$ level, which means that it was easy to insert the lumbar interbody fusion cage safely and accurately a. Fluoroscopic lateral image after extreme lateral interbody fusion showing more reduction of the $L 4$ vertebra $\mathbf{b}$

contraindicated because of the narrow anteroposterior width of the interbody space. Therefore, we developed the current surgical strategy of the reduction by the posterior PPS system in the lateral position prior to LLIF procedure.

Recently, it was reported that LLIF combined with posterior PPS for SL was associated with good clinical and radiological outcomes [8-17]. This combined procedure showed lower blood loss and muscle damage than other types of lumbar interbody fusion $[9,13,16$, 17]. Additionally, it has been indicated that this combined procedure is associated with a higher correction rate and better mechanical stability [12], higher bone union rate $[8,19]$, and lower complication rate $[8,11$, 19] than existing lumbar interbody fusion because a cage with a larger footprint is used. Good correction rates and clinical results were reported for isthmic SL by using the same procedure [14]. Therefore, this strategy may not only be a minimally invasive method but also an effective method of lumbar reconstruction surgery for SL. Moreover, another advantage was reported that performing LLIF with PPS with patients in the single lateral decubitus position saved time and money and improved operative efficacy $[20,21]$.

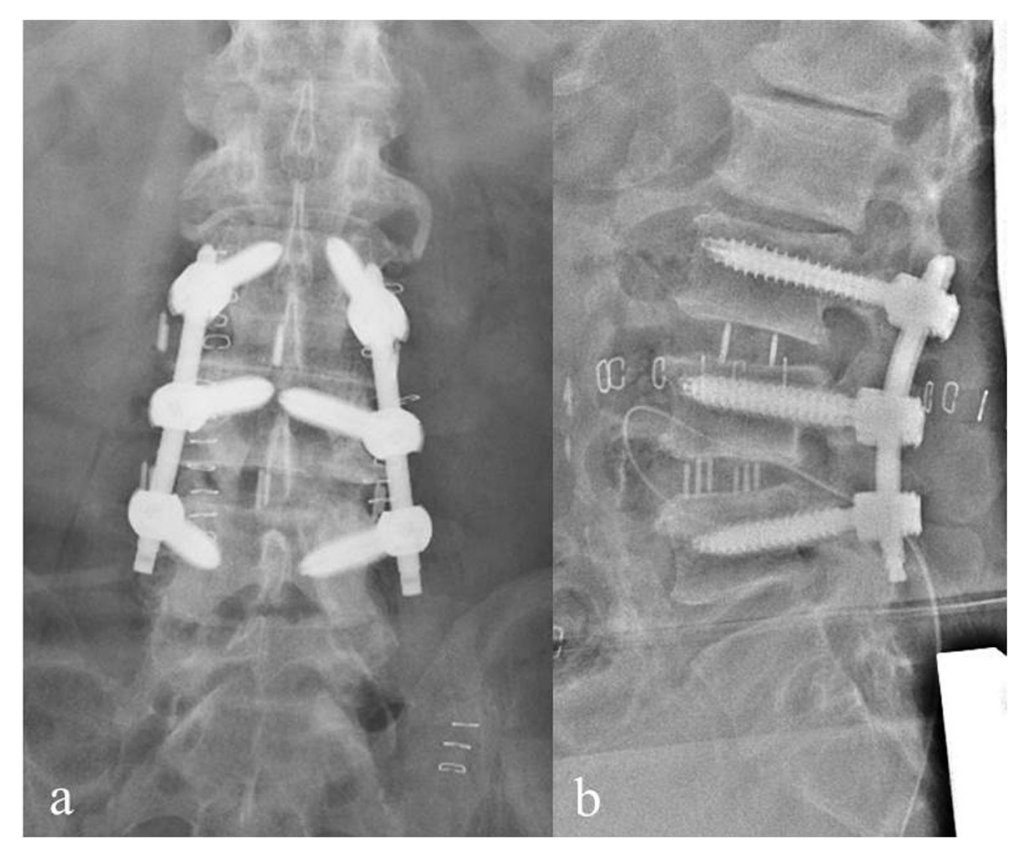

Fig. 6 Postoperative radiographic imaging. Postoperative radiographic images $\mathbf{a}$, b showing good alignment 
The main concern for surgeons was the occurrence of incidental anterior longitudinal ligament or neurological injury from inserting the cage into a narrow interbody space in patients with severe SL. The minimum anteroposterior width of the currently available cage for LLIF is $18 \mathrm{~mm}$ in Japan. Therefore, if the width was less than about $20 \mathrm{~mm}$, the surgeon cannot insert the cage using the conventional surgical procedure of LLIF theoretically. In some cases, the position may be slightly reduced. Nevertheless, it is considered dangerous to place the LIF cage first before reduction with PPS in patients with Meyerding grade II spondylolisthesis because the posterior nerves are very close to the retractor, and the appropriate range of cage placement is extremely narrow in the actual surgical field even if a slight reduction is obtained in the lateral position (particularly at L4/5 level). The surgeon must avoid both nerve injury and anterior longitudinal ligament injury, and hence, naturally, the surgeon prefers to have some extra space for safe and exact cage placement. We designed our novel surgical strategy to overcome this challenge.

The main limitation of this study was the small sample size and did not include a control group for statistical analysis of effectiveness of our method. The present six cases accounted for over $50 \%$ of all the cases who underwent XLIF combined PPS in the lateral position in our institution from 2018. However, in general, the rate of Meyerding grade II or higher is not very high in the SL. Second, the follow-up period may be short to assess the efficacy of this surgical strategy. Strong reduction for severe SL may lead to instability between the vertebrae. Fogel et al. [22] reported that a biomechanical test showed variability in construct stability in the sagittal plane of LLIF combined with PPS. LIFARL aims to achieve safe insertion of the LLIF cage with some reduction, and it is not intended for complete reduction. No cases of pseudarthrosis and implant-related complications occurred, however, it will be necessary to confirm the efficacy of this strategy in a larger number of patients with appropriate follow-up period in the future.

\section{Conclusion}

Conventional XLIF combined with PPS procedure for patients with SL of Meyerding grade II is contraindicated because of the narrow anteroposterior width of the interbody space. However, by using the LIFARL strategy, surgeons have an option to perform LLIF combined with posterior PPS accurately and safely. For patients with such pathology, the minimally invasive LIFARL strategy provides excellent clinical outcomes.

\section{Abbreviations}

LLIF: Lateral lumbar interbody fusion; XLIF: extreme lateral interbody fusion; PPS: Percutaneous pedicle screw; LIFARL: Lateral lumbar interbody fusion after reduction by the posterior percutaneous pedicle screw system in the lateral position; SL: Spondylolisthesis; PLIF: Posterior lumbar interbody fusion; CT: computed tomography; ODI: Oswestry disability index; VAS: Visual analog scale

\section{Acknowledgements}

None.

\section{Authors' contributions}

Author contributions to the study and manuscript preparation include the following. Conception and design: MT. Acquisition of data: MT. Analysis and interpretation of data: MT. Drafting the article: MT. Critically revising the article: AM and HY. Reviewed submitted version of manuscript: AM and HY. Approved the final version of the manuscript on behalf of all authors: MT. Statistical analysis: MT. Administrative/technical/material support: RT and MO, HH. Study supervision: HY

\section{Funding}

The authors declare that they have no sources of funding for research reported.

\section{Availability of data and materials}

All data generated or analyzed during this study are included in this published article.

\section{Ethics approval and consent to participate}

The study design was approved by the institutional review board of Wakayama Medical University before initiation of the study (approval number: 2809). The patients and/or their family members were informed that the patient data would be submitted for publication, and their written consent was obtained.

\section{Consent for publication}

All patients consented to publish personal data in an anonymized form. This manuscript has not been published or presented elsewhere in part or in entirety and is not under consideration by another journal.

\section{Competing interests}

The authors declare that they have no competing interest and no sources of financial supports.

Received: 13 May 2020 Accepted: 28 December 2020

Published online: 05 January 2021

References

1. Guigui P, Ferrero E. Surgical treatment of degenerative spondylolisthesis. Orthop Traumatol Surg Res. 2017;103:S11-20.

2. Ozgur BM, Aryan HE, Pimenta L, Taylor WR. Extreme Lateral Interbody Fusion (XLIF): a novel surgical technique for anterior lumbar interbody fusion. Spine J. 2006;6:435-43.

3. Foley KT, Gupta SK, Justis JR, Sherman MC. Percutaneous pedicle screw fixation of the lumbar spine. Neurosurg Focus. 2001;10:E10.

4. Proietti L, Perna A, Ricciardi L, Fumo C, Santagada DA, Giannelli I, et al. Radiological evaluation of fusion patterns after lateral lumbar interbody fusion: institutional case series. Radiol Med. 2020 Jul 11. doi: https://doi.org/ 10.1007/s11547-020-01252-5.

5. Parker RM, Malham GM. Comparison of a calcium phosphate bone substitute with recombinant human bone morphogenetic protein-2: a prospective study of fusion rates, clinical outcomes and complications with 24-month follow-up. Eur Spine J. 2017;26:754-63.

6. Rodgers WB, Gerber EJ, Patterson JR. Fusion after minimally disruptive anterior lumbar interbody fusion: analysis of extreme lateral interbody fusion by computed tomography. SAS J. 2010;4:63-6.

7. Castellvi AE, Nienke TW, Marulanda GA, Murtagh RD, Santoni BG. Indirect decompression of lumbar stenosis with transpsoas interbody cages and percutaneous posterior instrumentation. Clin Orthop Relat Res. 2014;472: 1784-91.

8. Khajavi K, Shen A, Hutchison A. Substantial clinical benefit of minimally invasive lateral interbody fusion for degenerative spondylolisthesis. Eur Spine J. 2015;24(Suppl 3):314-21.

9. Goodnough LH, Koltsov J, Wang T, Xiong G, Nathan K, Cheng I. Decreased estimated blood loss in lateral trans-psoas versus anterior approach to 
lumbar interbody fusion for degenerative spondylolisthesis. J Spine Surg. 2019;5:185-93.

10. Kono Y, Gen H, Sakuma Y, Koshika Y. Comparison of clinical and radiologic results of mini-open transforaminal lumbar interbody fusion and extreme lateral interbody fusion indirect decompression for degenerative lumbar spondylolisthesis. Asian Spine J. 2018;12:356-64.

11. Ohba T, Ebata S, Haro H. Comparison of serum markers for muscle damage, surgical blood loss, postoperative recovery, and surgical site pain after extreme lateral interbody fusion with percutaneous pedicle screws or traditional open posterior lumbar interbody fusion. BMC Musculoskelet Disord. 2017;18:415.

12. Ko MJ, Park SW, Kim YB. Correction of spondylolisthesis by lateral lumbar interbody fusion compared with transforaminal lumbar interbody fusion at L4-5. J Korean Neurosurg Soc. 2019;62:422-31.

13. Campbell PG, Nunley PD, Cavanaugh D, Kerr E, Utter PA, Frank K, et al. Short-term outcomes of lateral lumbar interbody fusion without decompression for the treatment of symptomatic degenerative spondylolisthesis at L4-5. Neurosurg Focus. 2018 Jan;44:E6.

14. Tamburrelli FC, Meluzio MC, Burrofato A, Perna A, Proietti L. Minimally invasive surgery procedure in isthmic spondylolisthesis. Eur Spine J. 2018; 27(Suppl 2):237-43.

15. Pereira EA, Farwana M, Lam KS. Extreme lateral interbody fusion relieves symptoms of spinal stenosis and low-grade spondylolisthesis by indirect decompression in complex patients. J Clin Neurosci. 2017;35:56-61.

16. Sembrano JN, Tohmeh A, Isaacs R. SOLAS Degenerative Study Group. Twoyear comparative outcomes of MIS lateral and MIS transforaminal interbody fusion in the treatment of degenerative spondylolisthesis: part I: clinical findings. Spine (Phila Pa 1976). 2016;41:S123-32.

17. Isaacs RE, Sembrano JN, Tohmeh AG. SOLAS Degenerative Study Group. Two-year comparative outcomes of MIS lateral and MIS transforaminal interbody fusion in the treatment of degenerative spondylolisthesis: part II: radiographic findings. Spine (Phila Pa 1976). 2016;41:S133-44.

18. Meyerding HW. Spondylolisthesis; surgical fusion of lumbosacral portion of spinal column and interarticular facets; use of autogenous bone grafts for relief of disabling backache. J Int Coll Surg. 1956;26:566-91.

19. Januszewski J, Beckman JM, Bach K, Vivas AC, Uribe JS. Indirect decompression and reduction of lumbar spondylolisthesis does not result in higher rates of immediate and long term complications. J Clin Neurosci. 2017:45:218-22.

20. Blizzard DJ, Thomas JA. MIS single-position lateral and oblique lateral lumbar interbody fusion and bilateral pedicle screw fixation: feasibility and perioperative results. Spine (Phila Pa 1976). 2018;43:440-6.

21. Hiyama A, Sakai D, Sato M, Watanabe M. The analysis of percutaneous pedicle screw technique with guide wire-less in lateral decubitus position following extreme lateral interbody fusion. J Orthop Surg Res. 2019;14:304.

22. Fogel GR, Turner AW, Dooley ZA, Cornwall GB. Biomechanical stability of lateral interbody implants and supplemental fixation in a cadaveric degenerative spondylolisthesis model. Spine (Phila Pa 1976). 2014;39:E1138-46.

\section{Publisher's Note}

Springer Nature remains neutral with regard to jurisdictional claims in published maps and institutional affiliations.

Ready to submit your research? Choose BMC and benefit from:

- fast, convenient online submission

- thorough peer review by experienced researchers in your field

- rapid publication on acceptance

- support for research data, including large and complex data types

- gold Open Access which fosters wider collaboration and increased citations

- maximum visibility for your research: over $100 \mathrm{M}$ website views per year

At $\mathrm{BMC}$, research is always in progress.

Learn more biomedcentral.com/submissions 